\title{
Geometry of singular space
}

\author{
SHING-TUng YAU
}

\begin{abstract}
This paper grew out from my talk for the inauguration of the Riemann Center in Hanover, Germany. In an attempt to understand what Riemann said in his famous paper in 1854 on the foundation of geometry, I propose a theory of geometry that hopefully can be used to understand singular space that may still satisfy the Einstein equation in a generalized sense. Some calculations are made in the appendix that allow us to perform Hodge theory, to calculate the heat kernel within our abstract framework.
\end{abstract}

1 The development of modern geometry that influenced our concept of space

2 Geometry of singular spaces

3 Geometry for Einstein equation and special holonomy group

4 Laplacian and construction of generalized Riemannian geometry in terms of operators

5 Differential topology of the operator geometry

6 Inner product on tangent spaces and Hodge theory

7 Gauge groups, convergence of operator manifolds and Yang-Mills theory

8 Generalized manifolds with special holonomy groups

9 Maps, subspaces and sigma models

10 Non-compact manifolds 
A.1 Tangent space and the spectrum of $C$

\section{The development of modern geometry that influenced our concept of space}

Bernhard Riemann (1826-1866) and his teacher C.F. Gauss (1777-1855) are no doubt the two great geometers who founded modern geometry.

The beautiful theory of Riemannian geometry has in effect changed our views of the concept of space which was introduced by the ancient Greek geometers.

It is fair to say that without this development, it would have taken many more years for Einstein (1879-1955) with helps from Grossman (1878-1936) and Hilbert (1862-1943) to accomplish the great theory of general relativity.

Riemann [13] initiated the concept of modern geometry through the following paper in 1854: Über die Hypothesen, welche der Geometrie zu Grunde liegen (The Hypotheses on which Geometry is Based). This paper is truly a spectacular work: Riemann had few works prior to inspire him or 
to provide guidance, with the exception of some bits of work of Gauss and some philosophical work of Herbart.

He felt that "the theorems of geometry cannot be deduced from the general notion of magnitude alone, but only from those properties which distinguished space from other conceivable entities, and these properties can only be found experimentally."

"We can only investigate their probability, and therefore a judgment as to the admissibility of extending them outside the limits of observation, in the realms of both the immeasurably great and the immeasurably small.

Either the physical reality on which space is founded must be a discrete variety, or else the foundation of its metric relation must be sought from outside source in the forces which bind together its elements ....

This takes us into the realm of another science - physics."

\section{Geometry of singular spaces}

I would like to reflect upon how we may think about geometry as a whole and what we can do in the future. The subject is connected with geometry, analysis and mathematical physics, and this is exactly what Riemann had in mind about 160 years ago when he thought about geometry.

He was very much concerned about the role of space in physics. As we saw in the above, he questioned what kind of concepts of space can be drawn from physics. One may note that his discussion of the heat conduction motivated him to give the definition of the curvature tensor.

Hence, I think any sensible motivation on the fundamental concept of space should be linked to the intuitions from physics of nature. We are facing a great challenge in this century on how to work out a concept of geometry that is capable to understand general relativity in the large and quantum physics in the small. There have been proposals on such geometry. The most outstanding one is the non-commutative geometry of Alain Connes [5].

I am not an expert of his work, as my taste in geometry is largely traditional geometry, as is motivated from intuitions coming from curvature, topology, physics and analysis, especially from the point of view of differential equations. However, what I said here may be considered as my first primitive step towards the understanding of a suitable version of quantum geometry. My goal is to understand geometry through operator theory where classical spacetime may disappear altogether. This view was already developed by Connes. Most likely much of what I presented here is known to him.

In any case, we may need to study the geometry of discrete spacetime and develop properties that may exhibit similarity with continuous spacetime. 
However, we need to review what we know about continuous spacetime first. Much of the continuous geometry has been developed since the time of Riemann and we like to preserve their key properties.

\section{Geometry for Einstein equation and special holonomy group}

In order to demonstrate what I propose, I shall focus on the theory of Einstein manifolds. The construction of the Riemannian version of a vacuum Einstein equation with a possible cosmological constant is still the most challenging problem in geometry and analysis. It is a problem in analysis as it provides a nice elliptic system in a suitable gauge. This system is non-linear and a good definition of weak solution of Einstein equation is needed. We shall find such a definition.

Only when the manifold has either a large group of symmetries or with special internal symmetry (or special holonomy group) do we know how to construct such Einstein manifolds.

Many complete and compact manifolds with special holonomy groups have now been constructed, thanks to the works of many geometers. It is remarkable that many of them are Einstein manifolds, i.e., their curvature tensor satisfies the equation of Einstein in the Riemannian setting.

Among manifolds with special holonomy group, we have a reasonable understanding of Kähler manifolds, Calabi-Yau manifolds and hyperkähler manifolds. However, we do not have a good control of manifolds with holonomy groups $G_{2}, \operatorname{Spin}(7)$ and $S p(1) S p(n)$. They are all Einstein manifolds with Ricci curvature equal to zero.

We need a theorem similar to the Calabi-Yau theorem [3, 14], which reduces problems in manifolds to algebro-geometric problems which can be solved by algebraic means. In classical general relativity, we are more interested in metrics with Lorentzian signature. In some stationary spacetimes such as the one described by the Kerr metric, there is a procedure called Wick rotation that can "analytically" continue the Einstein metric with Lorentzian signature to one with Riemannian signature. In an amazing manner, the singularities of spacetime disappear after Wick rotation. These manifolds play an important role in Gibbons-Hawking theory of quantum gravity [6]. Although the Wick rotation construction is done in an ad hoc manner, it is worthwhile to point out that the Wick rotated Kerr metric admits a non-trivial second order differential operator that commutes with Laplacian. I propose this to be a concept that generalises manifold with special holonomy group. It is still not known how to classify all Lorentzian 
manifolds with special holonomy groups. They may be important for general relativity. I shall not discuss manifolds with Lorentzian signature here.

When the holonomy group is a proper subgroup of the orthogonal group, these are special subspaces of the tensor product of tangent bundle, and the associated projection operators commute with the Laplacian that acts on functions and forms.

In particular, the eigenforms of the Laplacian have natural splitting coming from the projection operators. The theory of Hodge [8] made use of this powerful and natural splitting on harmonic forms, which account for topology of the manifold. It builds a bridge between topology and analysis.

I shall discuss how to generalize the concept of Riemannian geometry by using the Laplace operator. In this new setting, the holonomy group will be replaced by the graded ring of local operators that commute with the Laplacian.

\section{Laplacian and construction of generalized Riemannian geometry in terms of operators}

Most of the known Einstein manifolds are obtained by reduction of variables by group actions or by constructing manifolds with special holonomy groups, or by combining such constructions.

In such a process, we may have to handle spaces, which have singularities. The most common singularities that we can handle are orbifold singularities. However, their structures are not rich enough to describe problems in modern physics.

We need to enlarge the category of manifolds to allow manifolds with general singularities. However at the same time, we would like to keep the natural geometric operators to be well-defined on such singular spaces.

We propose to formulate a theory that replaces Riemannian manifolds by operators acting on a Hilbert space equipped with an algebra.

(i) On a compact manifold $M$, the Riemannian metric gives rise to a measure. If we normalize the total volume to be one, all these measures are equivalent to each other by a volume preserving diffeomorphism. Hence, we have a Hilbert space $H=L^{2}(M)$ and an algebra $\mathcal{A}$ of unitary operators defined by the group of measures preserving diffeomorphisms. (If we want to avoid the use of the measure, we can replace functions by half-densities.)

(ii) Within the Hilbert space $H$, we have a subalgebra $C$ of smooth functions that determines the differential structure of $M$. The 
Laplacian $L$ is a self-adjoint operator defined on $C$, which is local in the sense that for any $\varphi_{1}, \varphi_{2} \in C, \varphi_{1} \varphi_{2}=0 \Rightarrow \varphi_{1} L\left(\varphi_{2}\right)=0$.

We may not require $L$ to map $C$ into $C$ or $H$. However, we shall require $L(\varphi)$ to be a linear functional defined on $C$ and is symmetric in the following sense: for any $\varphi, \psi \in C$

$$
\langle L(\varphi), \psi\rangle=\langle\varphi, L(\psi)\rangle .
$$

(iii) The inner product $\left\langle\varphi,(-L)^{s} \varphi\right\rangle$ is positive on $\{\varphi \in C:\langle\varphi, 1\rangle=$ $0\}$. Its completions are Hilbert spaces that will be called $H_{s}$, we assume that the embedding $H_{1} \hookrightarrow H$ is compact.

The space of Riemannian metrics can be considered as the orbit space of the space of the triples $(H, C, L) \bmod \mathcal{A}$, the group of unitary operators defined by the group of measure preserving diffeomorphisms. Note that the algebra $\mathcal{A}$ is a subalgebra of the endomorphism ring of $C$. We would like to make sure this orbit space is Hausdorff and the concept of stable manifold in the sense of geometric invariant theory may be needed. In principle, we can therefore replace a Riemannian manifold by $(H, C, L)$, which satisfies the above properties.

In order for the triple to recover standard properties of Riemannian geometry, we shall make several assumptions.

(1) Compatibility of multiplication with inner product:

Hence,

$$
\left\langle f^{2}, 1\right\rangle=\langle f, f\rangle=\|f\|^{2} \quad\langle f g, h\rangle=\langle f, g h\rangle .
$$

We shall also assume that multiplication by $f \in C$ defines a bounded operator on $H$.

(2) The Cone of positive functions:

The Cone defined by taking $H-$ closure of $\left\{\sum_{i=1}^{k} \rho_{i}^{2}: \rho_{i} \in C\right\}$ will be called $H^{+}$.

Then for any element $\rho \in H$, there is a unique element $\rho^{+} \in H^{+}$ and $\rho^{-}$, so that

$$
\rho=\rho^{+}+\rho^{-}
$$

and $\left\langle\rho^{-}, g\right\rangle \leq 0$ for all $g \in H^{+}$.

If we define $\overline{H^{+}}=\left\{h \in H:\langle h, g\rangle \geq 0\right.$, for all $\left.g \in H^{+}\right\}$,

Then $H^{+} \subseteq \overline{H^{+}}, H^{+} \cdot \overline{H^{+}} \subseteq \overline{H^{+}}$and $-\rho^{-} \in \overline{H^{+}}$. 
It is easy to prove that

$$
\left\|\rho^{-}\right\|^{2}+\left\|\rho^{+}\right\|^{2} \leq\|\rho\|^{2}
$$

In any case, we shall assume that $L\left(\rho^{2}\right)-2 \rho L(\rho) \in \overline{H^{+}}$. We need this for proving that $\exp (t L)$ preserves $\bar{H}^{+}$. In order to define an inner product on the space of differentials, we assume further that for any set of elements $\left\{f_{i}, g_{i}\right\}$ in $C$, we have

$$
\sum g_{i} g_{j}\left[L\left(f_{i} f_{j}\right)-f_{j} L\left(f_{i}\right)-f_{i} L\left(f_{j}\right)\right] \in \overline{H^{+}}
$$

(3) The embedding from $H_{s}$ to $H_{s-1}$ are compact operators for all $s$. One can then show that the spectrum of $L$ is discrete and that it tends to infinity when $H$ is infinite-dimensional. Note that all the eigenfunctions of $L$ belong to $\cap_{s=0}^{\infty} H_{s}$.

(4) If $\lambda_{k}$ are the eigenvalues of $-L$, we assume that $\lambda_{k} \geq 0, \lim _{k \rightarrow \infty} k^{-\frac{2}{n}} \lambda_{k}$ exists and depends only on $\operatorname{vol}(M)$. This is Weyl's law in Riemannian geometry.

Tauberian theorems say that the Weyl law is equivalent to the statement

$$
\lim _{t \rightarrow 0} t^{\frac{n}{2}} \operatorname{tr} e^{t L}
$$

exists, and is equal to a number $a_{0}$ depending only on $\operatorname{vol}(M)$. We shall also assume the existence of

$$
a_{1}=\lim _{t \rightarrow 0} t^{-1}\left(t^{\frac{n}{2}} \operatorname{tr} e^{t L}-a_{0}\right)
$$

Note that in Riemannian geometry, $a_{1}$ is the total scalar curvature of the manifold $M$. The number $n$ seen above will be defined to be the dimension of the manifold. Hence, we shall consider $a_{1}$ as an action defined on the space of $(H, C, L) \bmod \mathcal{A}$. In this way, our generalized manifolds are Einstein if they are critical points of this functional.

An important example is a metric with $g_{i j}$ non-smooth but positive definite. We assume that $g_{i j}$ is bounded above and below by smooth Riemannian metrics. With low regularity on $g_{i j}$, one can make sense of $a_{1}$ and hence weak solution of Einstein equation. 


\section{Differential topology of the operator geometry}

With the algebra $C$, we can define the tangent bundle to be the space of derivations of $C$, which are also local operators defined on $H_{s}$ for all $s \geq 0$ such that for all $f \in H_{s}$,

$$
\|X f\|_{s} \leq a\|f\|_{s+1} .
$$

The tangent bundle is a module over $C$. The wedge product of the tangent vectors can be formed in the usual way. The dual spaces are differential forms. Given a function $f$ in $C$, we can define a differential form by $d f(X)=X(f)$.

If $d f_{1}, d f_{2}, \ldots, d f_{m}$ are linearly independent over $C$, then we expect that polynomials of them will produce enough functions to prove that the $k$ th eigenvalue of $L$ is not greater than $C k^{\frac{2}{m}}$ if certain scaling properties hold for $L$. In such cases, $m \leq n$.

It is quite likely that for an $n$-dimensional manifold, the differential of any $n$-distinct eigenfunctions with distinct non-zero eigenvalues are independent over $C$. It will be interesting to find conditions such that the analogous statement holds for the case of an operator manifold. That will mean that such a manifold has dimension $n$ iff the maximal $m$ such that $d f_{1} \wedge \cdots \wedge d f_{m} \neq 0$ is equal to $n$.

We shall assume that the space of derivation is a finite-dimensional module over $C$ and can be generated by finite number of derivations $\left\{X_{i}\right\}$ such that $\left[X_{i}, X_{j}\right]$ defined by

$$
\left[X_{i}, X_{j}\right](\varphi)=X_{i}\left(X_{j}(\varphi)\right)-X_{j}\left(X_{i}(\varphi)\right)
$$

are derivations satisfying (5.1)

Hence, $\left[X_{i}, Y_{j}\right]$ can be defined and they are derivations satisfying (5.1). This allows us to define exterior derivative on the forms which are dual space to the wedge product of tangent bundle.

The exterior algebra of cotangent bundle, which is the dual of the space of derivations, admits exterior differentiations in the standard manner. They can be called differential forms. Its cohomology can be considered as cohomology of the manifold.

The space of linear functional defined on the exterior algebra of cotangent bundle will be defined as currents in our geometry. It has a boundary operator dual to the exterior differentiation. We can then define the homology of the manifold accordingly. 
The de Rham forms can be defined by the $C$-modules generated by $d f_{1} \wedge \cdots \wedge d f_{m}$ and there is a natural exterior differentiation. The invariants associated to this complex should be interesting for singular manifolds. The cohomology defined by de Rham forms will be called de Rham cohomology. Since it is not clear that the dual of vector fields are spanned by the differential of functions, the cohomology defined by the exterior algebra of differential forms may be different from the de Rham cohomology.

\section{Inner product on tangent spaces and Hodge theory}

The space spanned by differential of functions can be written as $\omega=\sum_{i} g_{i} d f_{i}$. Its inner product can be defined so that for all $\rho \in C,\langle\rho \omega, \omega\rangle=$ $\sum_{i, j}\left\langle\rho g_{i} g_{j}, L\left(f_{i} f_{j}\right)-f_{i} L\left(f_{j}\right)-f_{j} L\left(f_{i}\right)\right\rangle$.

In Section 5, we assume this defines a positive-definite inner product on the space of differentials. In order for this expression to be well defined, we make the following assumption: if $\sum g_{i} X\left(f_{i}\right)=0$ for all derivation $X$, then for all $f$,

$$
\sum g_{i}\left(L\left(f f_{i}\right)-f L\left(f_{i}\right)-f_{i} L(f)\right)=0
$$

This simply says that if $\sum g_{i} d f_{i}(X)=0$ for all derivation $X$, then $\sum g_{i} d f_{i}$ is orthogonal to all other differentials.

The inner product on the forms should be compatible with the multiplication, i.e.,

$$
\langle g d f, d(h k)\rangle=\langle g d f, h d k\rangle+\langle g d f, k d h\rangle
$$

Therefore we require the following identity holds for $L$ :

$$
\begin{gathered}
L(f h k)=f L(h k)-h k L(f)+h L(f k)-f k L(h) \\
+k L(f h)-h f L(k) .
\end{gathered}
$$

The inner product on the tangent bundle can be defined by duality:

$$
\|X\|^{2}=\sup _{\|\omega\| \leq 1}|\langle X, \omega\rangle|^{2}
$$

where $\omega$ are differential one-forms. 
The inner product $\langle\omega, \omega\rangle$ may have kernel. We can mod out the kernel of the inner product to obtain a non-degenerate inner product.

For any vector field $X$ dual to $d f$ where $f \in C$, we define $\nabla_{X} \omega$ by the following rule:

$$
2 \nabla_{X} \omega=L(f \omega)-f(L \omega)-L(f) \omega
$$

This equation can be extended to $X$ which is dual to $\sum_{i} g_{i} d f_{i}$. The formula can be interpreted as an action of differential one-form on forms with arbitary degree, without referring to vector fields.

The operator $R_{i}$ defined by

$$
\left\langle R_{i} \omega_{1}, \omega_{2}\right\rangle=L\left\langle\omega_{1}, \omega_{2}\right\rangle-\left\langle L_{i}\left(\omega_{1}\right), \omega_{2}\right\rangle-\left\langle\omega_{1}, L_{i}\left(\omega_{2}\right)\right\rangle-\left\langle\nabla \omega_{1}, \nabla \omega_{2}\right\rangle
$$

will be called Bochner curvature operator.

Assuming $R_{i}$ defines a bounded operator on the $L^{2}$ space of $i$-forms, we form the Hilbert space obtained by completion of space of $i$-forms $\omega$ based on the norm $\|\nabla \omega\|^{2}$. It defines a compact embedding into the $L^{2}$ space of $i$-forms. This implies the harmonic $i$-forms are finite-dimensional. These statements will be discussed in more detail in the Appendix. Note that there may be kernel for $\|\nabla \omega\|^{2}$, namely, there may be forms $\omega$ so that $\nabla \omega=0$.

These forms are called parallel forms. Classically, existence of parallel forms give strong restriction on the geometric structure of the manifolds, such as special holonomy group or local product structure. We expect similar phenomena in our operator geometry. We shall come back to this later.

We can define $\mathcal{L}$ to be first-order operator $d \pm d^{*}$ mapping even forms to odd forms. It admits an adjoint $\mathcal{L}^{*}$. Then $\operatorname{tr}\left[\exp (t \mathcal{L})-\exp \left(t \mathcal{L}^{*}\right)\right]$ is constant in $t$ and gives rise to the index of $\mathcal{L}$ when $t \rightarrow \infty$. In classical geometry, they can be expressed as integrals of local differential forms defined by the curvature when $t \rightarrow 0$. This is the local index formula of Atiyah-Bott-Patodi [1]. It is therefore important to find good conditions for the existence of

$$
\lim _{t \rightarrow 0} \operatorname{tr}\left[\exp (t \mathcal{L})-\exp \left(t \mathcal{L}^{*}\right)\right]
$$

Perhaps they can be expressed in terms of the above operators $R_{i}$.

Note that as long as (4.5) and (6.3) hold, we can define the de Rham and Hodge theory without referring to vector fields. This may have advantages for discrete spaces. 


\section{Gauge groups, convergence of operator manifolds and Yang-Mills theory}

Given a vector field $X$, there is a function $\operatorname{div} X$ defined by

$$
\langle X(f), 1\rangle=-\frac{1}{2}\langle\operatorname{div} X, f\rangle,
$$

for all $f \in H$. Then

$$
\left\langle\left(X+\frac{1}{4} \operatorname{div} X\right) f, g\right\rangle=-\left\langle f,\left(X+\frac{1}{4} \operatorname{div} X\right) g\right\rangle .
$$

Therefore,

$$
X+\frac{1}{4} \operatorname{div} X
$$

is a skew adjoint operator and $\exp \left(X+\frac{1}{4} \operatorname{div} X\right)$ defines a unitary operator on $H$. It generates a gauge group acting on $(H, C, L)$. For most calculations, we can replace the group $\mathcal{A}$ by this gauge group.

Finite-dimensional vector bundles are projective modules over $C$ with finite rank. A metric on the vector bundle $V$ is simply a positive-definite symmetric pairing $\langle$,$\rangle on the projective module, which is linear over C$. A connection is a map $\nabla$ from the tensor product of the tangent bundle with the projective module to the projective module itself. It is linear over both variables, but linear over $C$ for the first variable.

(i) For $\rho \in C$ and $W \in V$,

$$
\nabla_{X}(\rho W)=X(\rho) W+\rho \nabla_{X} W
$$

$$
\nabla_{X}\left\langle W_{1}, W_{2}\right\rangle=\left\langle\nabla_{X} W_{1}, W_{2}\right\rangle+\left\langle W_{1}, \nabla_{X} W_{2}\right\rangle
$$

For each vector field $X, \nabla_{X}$ defines an operator from the vector bundle into itself. It has an adjoint $\nabla_{X}^{*}$. Hence, we can define an operator

$$
\Delta=\sum_{e_{i}} \nabla_{e_{i}}^{*} \nabla_{e_{i}}
$$

where $\left\{e_{i}\right\}$ form an orthonormal basic for the space of vector fields. The operator $\Delta$ is independent of the choice of the basis, but depends on the connection. 
Note that in this discussion, we can replace $X$ by differential one-form. In that case, $X(\rho)$ is simply the inner product of the one-form with $d \rho$.

We shall assume that $t^{\frac{n}{2}} \exp (t \Delta)$ has an expansion $a_{0}+a_{1} t+a_{2} t^{2}+$ $o\left(t^{2}\right)$ when $t$ is small. The number $a_{2}$ can be considered as action on the space of connection. Classically when we fix the metric on the base manifold, this gives the Yang-Mills action plus some square integrals of the curvatures of the metric. Hence, we can define Yang-Mills connections for vector bundles.

It is natural to introduce de Rham theory with coefficient in a vector bundle, and this can be done by using the connection on the vector bundle. Index theory can be also developed.

In order to define distance between $\left(H, C, L_{1}\right)$ and $\left(H, C, L_{2}\right)$, we replace the triple by $\left(H, C, e^{t L_{1}}\right)$ and $\left(H, C, e^{t L_{2}}\right)$ respectively and we define their square distance by

$$
\int_{0}^{\infty} t^{\frac{n}{2}} \operatorname{tr}\left(e^{t L_{1}}-e^{t L_{2}}\right)^{2} d t
$$

The distance between $\left(H, C, e^{t L_{i}}\right) \bmod \mathcal{A}$ is obtained by taking the distance between the orbits of $\mathcal{A}$ acting on $e^{t L_{i}}$,

$$
\min _{B \in \mathcal{A}} \int_{0}^{\infty} t^{\frac{n}{2}} \operatorname{tr}\left(B^{-1} e^{t L_{1}} B-e^{t L_{2}}\right)^{2} d t .
$$

It is perhaps useful to replace $t$ by complex number if we are interested to look into the Schrödinger operator, we look for operators $L$ so that $\exp (t L)$ can be analytically continued to a disk $|t| \leq a$. The square distance can be defined by

$$
\min _{B \in \mathcal{A}} \int_{|t| \leq a}|t|^{n} \operatorname{tr}\left\|\exp \left(t L_{1}\right)-\exp \left(t L_{2}\right)\right\|^{2} d t d \bar{t}
$$

In either distance, the limiting element can be considered as a singular Riemannian manifold. The advantage of the definition of such a singular manifold is that we have naturally defined geometric operators associated with them.

The distance between operator manifolds with the same algebra $C$ was defined by the above definitions. However, when the algebra $C$ is different, we shall use the following definition:

Given two operator manifolds $\left(H_{1}, C_{1}, L_{1}\right)$ and $\left(H_{2}, C_{2}, L_{2}\right)$, we look for another operator manifold $\left(H_{3}, C_{3}, L_{3}\right)$ such that there are bounded linear maps from $H_{3}$ to $H_{1}$ and $H_{2}$, which induce surjective homomorphisms from $C_{3}$ to $C_{1}$ and $C_{2}$, respectively. We also assume there are sections of these 
maps, i.e., bounded linear maps $F_{1}$ and $F_{2}$ mapping $C_{1}$ and $C_{2}$ back to the $C_{3}$ so that the composite maps are identity maps.

For any orthonormal basis of $H_{1}$ consisting of vectors $\left\{\theta_{i}\right\}$ in $C_{1}$, we lift them to $C_{3}$ by $F_{1}$ and then map to $C_{2}$ to obtain vectors in $C_{2}$ which we then operate by $\exp \left(t L_{2}\right)$. Similarly, we can let $\exp \left(t L_{1}\right)$ operate on $\theta_{i}$ and then lift them up to $C_{3}$ by $F_{1}$ and project them to $C_{2}$. Then we take the difference of these two set of vectors. We take their square norms in $\mathrm{H}_{2}$ and sum them up with respect to $i$. Then multiply with $t^{n / 2}$ and integrate with respect to $t$. Finally, we minimize the choices among all $H_{3}$ and the maps $F_{1}$ and $F_{2}$. The end result will be the square distance between $\left(H_{1}, C_{1}, L_{1}\right)$ and $\left(H_{2}, C_{2}, L_{2}\right)$ :

$$
\min _{H_{3}, F_{1}, F_{2}} \int_{0}^{\infty} t^{\frac{n}{2}} \sum_{i}\left\|\exp \left(t L_{2}\right) F_{1}\left(\theta_{i}\right)-F_{1}\left(\exp \left(t L_{1}\right) \theta_{i}\right)\right\|_{H_{2}}^{2} d t
$$

On the other hand, if we fix $H$ and the algebras $C_{1}, C_{2}$ are subalgebras of $H$, we can define a distance between $C_{1}$ and $C_{2}$ in the following way: take any two elements $\varphi_{1}, \varphi_{2}$ in $C_{1}$ with $H_{1}$-norm equal to one, project it into two elements $\overline{\varphi_{1}}$ and $\overline{\varphi_{2}}$ in the closure of $C_{2}$ with respect to the $H_{1}$-norm. Then the algebra norm of the projection $P$ in $\operatorname{Hom}\left(C_{1}, C_{2}\right)$ can be defined to be

$$
\begin{aligned}
& \sup \left\{\left\|\varphi_{1} \varphi_{2}-\overline{\varphi_{1}} \overline{\varphi_{2}}\right\|_{H}:\right. \\
& \left.\quad \forall \varphi_{1}, \varphi_{2} \in C_{1}, H_{1} \text { norm of } \varphi_{1} \text { and } \varphi_{2} \text { equals } 1\right\}
\end{aligned}
$$

In the other direction, we can define the algebra norm of the projection from $C_{2}$ to $C_{1}$. Adding these two norms together gives rise to a distance between $C_{1}$ and $C_{2}$.

In the above discussions, I did not discuss the Dirac operator as its existence requires vanishing of the second Stiefel-Whitney class, which is not defined over real number. An easy way to go around this is to start out from the Dirac operator instead of the Laplacian acting on functions. We shall come back to this topic later.

\section{Generalized manifolds with special holonomy groups}

Special holonomy group gives rise to projection operators acting on the tangent bundle or subspaces of tensor product of copies of tangent bundle and cotangent bundles. These operators are local and commute with the Laplacian. 
From this point of view, it is therefore natural to generalize the concept of manifolds with special holonomy group to these manifolds whose Laplacian has non-trivial local commuting or anti-commuting local operators.

In this regard, it is natural to ask the following question: If $\left\{\varphi_{i}\right\}$ is the orthonormal basis of eigenfunctions of $L$, then for a sequence of positive numbers $\left\{a_{i}\right\}$ such that $a_{i} \sim i^{\frac{m}{n}}$, when will the operator $\sum a_{i} \varphi_{i} \otimes \varphi_{i}$ define a local operator?

The order of the operator will be called $m$. These operators form a graded algebra by itself. It will be interesting to develop a theory to understand those manifolds where this graded algebra is large.

This question is interesting even when we deal with classical Riemannian geometry. When the manifold is the Riemannian Kerr metric, there is a nontrivial second-order operator commute with the Laplacian.

The generalized manifold is said to have symplectic structure if there is a skew-symmetric pairing $\omega(X, Y)$ on the space of vector fields and that $d \omega=0$. In this case, for any $f \in C$, we can associate a vector field $X_{f}$ by

$$
d f(Y)=\omega\left(X_{f}, Y\right)
$$

for all $Y$.

The Poisson bracket between two functions $f, g$ are defined by $\{f, g\}$, so that

$$
X_{\{f, g\}}=\left[X_{f}, X g\right] .
$$

The cycles defined by an ideal $I$ in $C$ will be Lagrangian if $I$ is invariant under the Poisson bracket. In this way, we can define Lagrangian cycles with singularities.

We shall define Kahler manifold to be these manifolds admitting an almost complex structure $J$ which acts on the tangent bundle which satisfies $J^{2}=-\mathbb{I}$ and also commute with the action of Laplacian on differentia forms. As a consequence, the de Rham cohomology will have the Hodge structure and most of the standard theory will go through.

\section{Maps, subspaces and sigma models}

The idea of using space of maps (worldsheets) from Riemann surfaces to determine structures of manifolds, as was done in string theory, has led to many interesting properties of manifolds. This is the sigma model of the manifold. It gives rise to conformal field theory if spacetime has special property. 
The idea of associating a "conformal field theory" to manifolds with special holonomy group has contributed immensely to understanding the study of such manifolds.

One of the major achievements is the discovery of the concept of mirror symmetry [15], where many interesting questions can be understood through duality. It arises from conformal field theory. Hence, it will be interesting to associate a conformal field theory to our singular space with special structure.

Subspaces of our abstract manifold can be defined by closed ideals of $C$ in the $H_{s}$ topology. Then there is naturally defined induced Laplacian acting on the quotient algebra and we can also define mappings of manifolds. We shall explain this in the following.

A point on the manifold is defined by the maximal ideal of functions in $H_{s}$, which vanishes at that point. Here $H_{s}$ are the closure of the algebra $C$ in the norm $\left\langle\varphi,-L^{s} \varphi\right\rangle$. A closed subset $E$ is defined by some closed ideal $I$ of functions in $C$ vanishing on this subset. The closure of $I$ in $H_{s}$ will be called $I_{s}$. We choose $s$ large enough, so that functions in $H_{s}$ are continuous, by Sobolov embedding theorem. The quotient space $H_{t} / I_{t}$ and $H_{s} / I_{s}$ admit natural inner products inherited from $H_{t}$ and $H_{s}$, respectively. They give rise to a new triple $\left(H_{t}^{\prime}, H_{s}^{\prime}, L_{t, s}^{\prime}\right)$ because the inner product on $H_{s} / I_{s}$, when compared with the inner product on $H_{t}^{\prime} / I_{t}$, defines a self-adjoint operator $L_{t, s}^{\prime}$. We can consider the $\lim \left(L_{t, s}^{\prime}\right)^{\frac{1}{s}}$ when $s \rightarrow \infty$ and define it to be our Laplacian $L^{\prime}$. Hence we have a new triple $\left(H_{t}^{\prime}, \bigcap_{s} H_{s}^{\prime}, L^{\prime}\right)$. The derivations of $H_{s}^{\prime}$ can be obtained by derivations of $C$ that preserve the ideal $I$.

The new triple can be considered as the triple associated to the closed subset $E$. Note that the ideal $I$ carries more information than the set $E$ itself. The set $E$ may be zero set of different ideals and the geometry can be different for different ideals. It would be useful to understand the spectral resolution of $L^{\prime}$ when the closed set is complicated. An important question is when the dimension of this closed subset, by looking at the trace of $\exp \left(t L^{\prime}\right)$, is related to the Hausdorff dimension of the subset or some other related definitions of dimension.

There are natural morphisms between triples which can be considered as generalization of maps from manifolds to each other. An important consideration is the sigma model where we consider maps from two-dimensional surfaces to the manifold.

Two-dimensional surfaces are those triples where the spectrum of the operator grows linearly. It will be interesting to prove the following possible generalization of classical uniformization theorem: two-dimensional triple is conformally isomorphic to a triple formed by a compact surface whose metric 
has constant curvature. Conformal means that the operator is the same as the Laplacian of the metric with constant curvature, up to multiplication by a function.

Sigma model considers the space of maps from our given triple to space of all triples defined by compact surfaces. It is a homomorphism mapping the algebra from one to another.

The homomorphism from $C_{2}$, the space of functions defined on the manifold triple to $C_{1}$, the space of functions defined on the surface triple, gives rise to a subspace of $C_{1}$, which is the image of $C_{2}$ under the homomorphism. This subspace is the quotient of $C_{2}$ by an ideal $I$. We can define the inner product on this subspace by taking the orthogonal complement of $I$ in $C_{2}$ by the $H_{1}$ inner product on $C_{2}$. Comparing the original inner product from the surface triple and this new inner product, we can define a self-adjoint operator whose trace defines the energy of the map. Harmonic maps are defined to be the critical point of this energy.

In general, when we have a homomorphism from $C$ of one manifold triple to the algebra of another one, we say that there is a smooth map from the second manifold to the first manifold. If there is a homomorphism from the algebra $H_{s}$ of $M_{1}$ defined by completion of $C_{1}$ using $H_{s}$ norm, to the $H_{t}$ of $M_{2}$ defined by completion of $C_{2}$ using $H_{t}$ norm, we can say the map is $t-s$ regular. The map is called embedding if the homomorphism induces isomorphism from $C_{2}$ to $C_{1}$ modulo the kernel of the homomorphism. It is called immersion if for each maximal ideal of $C_{2}$, there is an element $f$ not in that ideal so that $f$ multiplies $C_{1}$ is isomorphic to the algebra defined by $f$ multiplies $C_{2}$ mod the kernel of the homomorphism.

The space of vector fields in a manifold triple form a Lie algebra under Lie bracket. A vector field $X$ defines an action on the space of vector fields by taking Lie bracket. It is called Killing vector field if this operator is skew symmetric. Naturally the Jacobian identity allows us to prove that the space of Killing fields form a sub Lie algebra. This Lie algebra should be finitedimensional if Sobolev inequality holds on our manifold triple. Moreover, it should be the Lie algebra of the group of automorphism of the manifold triple.

Given a map from one manifold triple $M_{2}$ to another triple $M_{1}$, we can have two Lie algebra of vector fields on $M_{2}$ : namely the algebra of functions defined on $M_{2}$ that comes from the algebra of $C_{1}$ mod kernel ideal, need not be the same as the algebra of functions on $M_{2}$, unless we have an embedding. The space of vector fields in $M_{1}$ that preserves the kernel ideal defines a new Lie algebra; here we identify two vector fields if their action on any functions 
on $C_{1}$ differs by an element in the kernel ideal. The difference of these two Lie algebras measures the "singularity of the map".

\section{Non-compact manifolds}

To model after non-compact manifolds, we replace the algebra $C$ by smooth functions with compact support. The spectrum of $L$ may not tell us too much. We have to look at the distribution:

$$
\rho \longrightarrow t^{n / 2} \operatorname{tr}(\rho \exp (t L))=a_{0}(\rho)+a_{1}(\rho) t+\cdots
$$

When $t \rightarrow 0$, we can obtain the heat coefficient with weight $\rho$. In particular, we obtain the integral of $\rho R$, where $R$ is the scalar curvature. Hence, $R$ is a well-defined distribution.

Einstein manifolds are the triples, which are stationary with respect to the action $a_{1}(\rho)$ for all $\rho$. (Cosmological constant is obtained by the action $a_{1}(\rho)-\alpha\langle\rho, 1\rangle$, where $\alpha$ is the cosmological constant.)

All the heat coefficients can be recovered as distribution.

\section{Discrete spaces}

As pointed out by Riemann, the basic concept of space may consist of discrete objects. The formulation discussed in the first part can work for discrete space also.

A very important discrete space is simply a graph that consists of a bunch of vertices and edges joining them. We can use graphs to approximate singular spaces.

The study of the geometry of graphs can be fruitful. We need to implement structures over graphs so that it reflects geometry of continuous space. We can construct exactly the same triple as we did in the continuous case.

When we consider space of functions defined on the vertices of the graph, the multiplication of them can be allowed to spread out in a neighbourhood of each vertex. The tangent vectors are linear combinations of edges which act on these functions as derivations. Practically, all the structures mentioned above can be carried over.

It turns out that many interesting structures can be defined based only on the combinatorial structure of the graph that resembles continuous geometry. 
For example, A. Grigor'yan, Yong Lin, Y. Muranov and myself [7] have found a certain type of graph cohomology that resembles de Rham cohomology which need not be trivial even when the degree of the cohomology is big.

There are also several definitions of Ricci curvature for graphs $[2,4,9$, $10,12]$ and we can develop theorems parallel to theorems in Riemannian geometry.

There is a natural operator associated to the graph: the graph Laplacian acting on a function is obtained by averaging the function in a suitable manner.

Moreover, there are operators acting naturally on de Rham forms as I mentioned above. They are all important operators that can provide invariants for the graph. We can therefore study Hodge theory on graphs. We may like to look at local operators that commute with such operators as was mentioned in the above. As a result, we can provide special structures over the combinatorial part of the graph.

\section{Conclusion}

We have proposed new structures over continuous spaces and discrete spaces that allow us to discuss them in the same setting. They provide many interesting questions for classical geometry.

On the other hand, there are not enough physical intuitions behind the construction. While many interesting geometrical and combinatorial problems have already appeared, we are still a long way to understanding quantum geometry: a geometry that can incorporate quantum mechanics in the small and general relativity in the large.

Here are some open problems:

1. Under what condition can a manifold triple be embedded into Euclidean space where the algebra $C$ is the smooth functions with compact support?

2. Under what condition does the automorphism group of the manifold triple form a Lie group with the Lie algebra given by Killing vector fields?

3. Develop the whole theory based on Clifford algebra and derive the formula for Dirac operators with coefficients on bundles. Furthermore, find the index of the operators in terms of the asymptotic coefficients of the heat kernel on forms. Under what the conditions are the operators 
Fredholm and the index is invariant under deformations of the manifold triples. Study $K$-theory of the manifold triples.

4. In Section 10, we mention that we can define scalar curvature of the manifold triple as a distribution. If it is zero, what can we say about the manifold triple. Should the corresponding $\hat{A}$ genus vanish, when it is suitably defined.

5. Study sigma model for the manifold triple and derive the anomaly equations. Then construct conformal field theory for these manifold triples. Study manifold triple with boundary, so it should be a pair of manifold triples. Also study the cobordism theory and the singular cohomology of the manifold triples.

6. Study the question of surgery on such manifold triple. When the manifold triple is Einstein, what kind of obstruction we may find in terms of index of operators and suitably defined fundamental group of the manifold triple.

7. For cotangent space, one can define a natural symplectic structure and that should be useful for quantization.

\section{Acknowledgment}

I would like to thank the following persons for discussions and helps: $\mathrm{Mu}-$ Tao Wang, Richard Schoen, Bong Lian, Si Li, Andrew Strominger, Hao Xu and Jie Zhou.

\section{Appendix A}

In this appendix, we shall clarify and provide some general conditions for the operators to satisfy our hypothesis.

\section{A.1. Tangent space and the spectrum of $C$}

On a manifold, the set of functions vanish at one point form a maximal ideal $m$ in $C$. If $C$ is the space of smooth functions, the space of maximal ideals can be identified with the manifold. Each element $f$ in $C$ can be considered as a function defines on the space of maximal ideals by simply assign the value $f(m)$ to $m$ where $f(m)$ is the unique scalar multiple of the constant function 1 such that $f-f(m)$ belongs to $m$. There is a topology and a measure defined on the space of maximal ideals by requiring all the 
functions come from $C$ to be continuous. The inner product of $H$ defines integration on this space. For any open set $\Theta$ containing $m$, we assume that $\cap m^{\prime} \subset m^{i}$ for $m^{\prime} \subset \Theta$ for any $i>0$. We shall assume that intersection of all maximal ideals is trivial so that the map $f \longrightarrow\{f(m)\}$ is one to one.

The space $m / m^{2}$ can be considered as the cotangent space at the point represented by $m$. However, we need to modify this definition to obtain a cotangent space with an inner product.

The space $m^{2}$ is in general not closed in $H_{s}$ topology and its closure can be equal to closure of $m$ unless $s$ is large enough. We shall assume such an $s$ exists such that $\bar{m}_{s}^{2}$, the closure of $m^{2}$ in space $H_{s}$ is a proper closed subideal of $\bar{m}_{s}$, where $\bar{m}_{s}$, the closure of $m$ in $H_{s}$. The inner product of $H_{s}$ give rise to an inner product on $\bar{m}_{s}$ and hence on $\bar{m}_{s} / \bar{m}_{s}^{2}$, as quotient space of $\bar{m}_{s}$ by a closed subspace $\bar{m}_{s}^{2}$. There is an orthogonal splitting of $\bar{m}_{s}$ into $F_{s} \oplus_{s} \bar{m}_{s}^{2}$. The space $F_{s}$ can be embedded into the original Hilbert space $H$. We define $\lim _{s \rightarrow \infty} F_{s}$ with the induced metric from $H$ to be our cotangent space.

Note that the elements in the tangent bundle, namely the derivations of $C$, define linear maps on $\bar{m}_{s} / \bar{m}_{s}^{2}$. In fact, if $f_{i} g_{i}, h_{i}$ are in $\bar{m}_{s}$, then for any derivation $X, X\left(f+\sum_{i} g_{i} h_{i}\right)$ mod $\bar{m}_{s}$ gives a real number $X(f)$ independent of choice of $g_{i}$ or $h_{i}$. (Note that $X$ is a bounded operator on $H_{s}$ for all s.) This is because $\sum_{i} g_{i} X\left(h_{i}\right)+\sum_{i} h_{i} X\left(g_{i}\right)$ is an element of $\bar{m}_{s}$. Hence, $X(f)$ is well defined and linear. Conversely, if for each $\bar{m}_{s}$, we have a linear functional $I_{\bar{m}_{s}}$ on $\bar{m}_{s} / \bar{m}_{s}^{2}$, we can define a derivation $X$ by mapping each $f \in C$ to $X(f)=I_{\bar{m}_{s}}(f-f(m))$, where $f(m)$ is the unique scalar multiple of the constant function I so that $f-f(m) \in m$.

Since $(f-f(m))(g-g(m)) \in m^{2}, X[(f-f((m))(g-g(m)]=0$ and one proves easily that $X(f g)\left|d_{m}=f(m) X(g)\right|_{m}+\left.g(m) X(f)\right|_{m}$, we claim that the derivation $X$ defined in this way is a local operator, i.e., if $f, g \in C$ and $f g=0$, then $g X(f)=0$. This can be seen as follows: suppose $g \notin m$, then by continuity, $g \notin m^{\prime}$ for all maximal ideal in a neighbourhood of $m$. Since $f g \in m^{\prime}, f \in m^{\prime}$ for all $m^{\prime}$ near $m$. This implies that $f \in m^{2}$. Hence, $I_{m(f)=0}$ and $g X(f)=0$.

In order for $X$ to be a derivation, we need to assume

$$
\left\|I_{m}(f-f(m))\right\|_{s} \leq a_{s}\|f\|_{s+1},
$$

where $a_{s}$ is independent of $f$.

For $f \in C$, we can project $f$ to $\bar{m}_{s} / \bar{m}_{s}^{2}$ by taking away the constant term $f(m)$. This element defines $d f$ as a bounded linear functional on the space of derivation. For any $f_{i}$ and $g_{i}$ in $C, \sum_{i} g_{i} d f_{i}$ defines a map from $m$ to $\bar{m}_{s} / \bar{m}_{s}^{2}$. It maps $m$ to $\sum g_{i}(m)\left(f_{i}-f_{i}(m)\right)$. Hence, it has a norm $\left\|\sum g_{i} \cdot d f_{i}\right\|_{m}^{2}$ and 
we can integrate it over the space of $m$. In this way, we have another inner product for the space of differentials.

\section{A.2. Eigenvalues by method of variational calculus}

We shall see that the asymptotic of eigenvalues of $-L$ so that the Weyl law $\lim _{k \rightarrow \infty} k^{-\frac{2}{n}} \lambda_{k}$ holds is related to covering properties of the space.

We assume that there are (plenty of) non-negative functions $\rho_{i}$ such that

$$
\sum_{i=1}^{k} \rho_{i}^{2}=1
$$

and there are positive constants $\lambda\left(\rho_{i}\right)$ such that the following Poincaré inequality holds:

$$
\frac{1}{2}\left\langle\rho_{i}^{2}, L\left(\varphi^{2}\right)\right\rangle-\left\langle\rho_{i}^{2} \varphi, L \varphi\right\rangle \geq \lambda\left(\rho_{i}^{2}\right)\left\langle\rho_{i}^{2} \varphi, \varphi\right\rangle
$$

for all $\varphi$ such that $\left\langle\rho_{i}^{2}, \varphi\right\rangle=0$. In particular, if $\varphi$ is perpendicular to $\rho_{i}^{2}$ for all $i=1, \ldots, k$, we can sum the above inequality to obtain $-\langle\varphi, L \varphi\rangle \geq$ $\min _{i} \lambda\left(\rho_{i}^{2}\right)\langle\varphi, \varphi\rangle$.

The $\max -\min$ principle characterization of eigenvalues of $L$ then says that

$$
\lambda_{k+1} \geq \min _{i} \lambda\left(\rho_{i}^{2}\right)
$$

Since we can vary the choice of $\rho_{i}^{2}$, we see that

$$
\lambda_{k+1} \geq \bar{\lambda}_{k+1}:=\max _{\rho_{i}} \min _{i} \lambda\left(\rho_{i}^{2}\right)
$$

where $\rho_{i}$ satisfies (A.1) and (A.2).

In Riemannian geometry, $\rho_{i}^{2}$ can be chosen to be characteristic functions of balls in $M$ which cover $M$ and the number of overlap of the balls are bounded by a constant depending on the dimension. The number of such balls are $k \backsim\left(\frac{1}{r}\right)^{\frac{1}{n}} \operatorname{vol}(M)$ where $n=\operatorname{dim} M$ and $\lambda\left(\rho_{i}\right) \backsim \frac{1}{r^{2}} \backsim\left(\frac{k}{\operatorname{vol}(M)}\right)^{\frac{2}{n}}$. Note that $r^{2}$ reflects the order of $L$ is chosen to be two. Hence, $\lambda_{k+1} \gtrsim$ $\left(\frac{k}{\operatorname{vol}(M)}\right)^{\frac{2}{n}}$.

As for upper bound of $\lambda_{k+1}$, we consider $\rho_{i}$ such that $\left\langle\rho_{i} \rho_{j}\right\rangle=0$ for $i \neq j$ and $\left\langle\rho_{i}, \rho_{i}\right\rangle=1$. Then for $\psi=\sum a_{i} \rho_{i}$,

$$
-\langle\psi, L \psi\rangle=-\sum a_{i}^{2}\left\langle\rho_{i}, L \rho_{i}\right\rangle=\min _{i}\left[-\left\langle\rho_{i}, L \rho_{i}\right\rangle\right]\langle\psi, \psi\rangle .
$$


Hence, $\lambda_{k+1} \leqslant \min _{i}\left(-\left\langle\rho_{i}, L \rho_{i}\right\rangle\right)$. In the case of Riemannian geometry, we choose $\rho_{i}$ to be the first eigenfunction of the Dirichlet problem of balls that are disjoint. Hence, $-\left\langle\rho_{i}, L \rho_{i}\right\rangle \sim\left(\frac{1}{r}\right)^{2}$ and $k \backsim\left(\frac{1}{r}\right)^{\left(\frac{1}{n}\right)} \operatorname{vol}(M)$. Therefore, $\lambda_{k+1} \leqslant C^{\prime}\left(\frac{k}{\operatorname{vol}(M)}\right)^{\frac{2}{n}}$.

In general, we allow choice of $\rho_{i}$ so that $\left\langle\rho_{i}, \rho_{j}\right\rangle=\delta_{i j}$, we define

$$
\overline{\bar{\lambda}}_{k+1}=\min _{\rho} \min _{i}\left[-\left\langle\rho_{i}, L \rho_{i}\right\rangle\right] \text {. }
$$

Then

$$
\bar{\lambda}_{k+1} \leq \lambda_{k+1} \leq \overline{\bar{\lambda}}_{k+1},
$$

where $\bar{\lambda}_{k+1}$ is defined in equation (A.10). If we assume that

$$
\lim _{k \rightarrow \infty} k^{-\frac{2}{n}} \bar{\lambda}_{k}=\lim _{k \rightarrow \infty} k^{-\frac{2}{n}} \overline{\bar{\lambda}}_{k}
$$

which depends only on $\operatorname{vol}(M)$, as is similar to the case of smooth manifolds, the Weyl law holds and $n$ would be the dimension of our space.

Theorem A.1. If the triple is defined by a Riemannian manifold whose metric tensor $\sum g_{i j} d_{x}^{i} d_{x}^{j}$ is only measurable, but bounded between two smooth Riemannian metric, then the dimension of the triple as defined by spectrum of the Laplacian is the dimension of the manifold.

\section{A.3. Weak maximum principle for heat equation}

Given any element $\rho \in H, \rho_{t}=\exp (t L) \rho$ will satisfy the heat equation:

$$
\left\{\begin{array}{l}
\frac{d \rho_{t}}{d t}=L\left(\rho_{t}\right), \\
\lim _{t \rightarrow 0} \rho_{t}=\rho .
\end{array}\right.
$$

Since $-L$ is a positive operator, one can easily prove that for all $i>0$

$$
\left\langle\rho_{t},(-L)^{i} \rho_{t}\right\rangle<\infty
$$

and hence $\rho_{t} \in H_{i}$ for all $i$. In classical manifold theory, $\cap_{i=1}^{\infty} H_{i}$ are smooth functions. While $C \subset \cap_{i=1}^{\infty} H_{i}$ in general, it will be useful to find conditions so that $C=\cap_{i=1}^{\infty} H_{i}$. For classical geometry, this is a consequence of Sobolev embedding theorem. In the following we shall relax the equation (A.8) to $\frac{\partial \rho}{\sigma t}-L(\rho) \varepsilon \overline{H^{+}}$and derive consequences. 
Suppose the initial data $\rho \in H^{+}$, i.e., $\left\langle\rho, f^{2}\right\rangle>0$ for all $f \neq 0 \in H$. We would like to demonstrate that the same inequality holds true for all $t$. This may be considered as a weak maximum principle.

In order to achieve this, we make the assumption that for any $f \in H_{1}$, which is not a multiple of 1 ,

$$
0 \neq L\left(f^{2}\right)-2 f \cdot L(f) \in H^{+} .
$$

Then

$$
\frac{d}{d t}\left\langle\rho, f^{2}\right\rangle=2\left\langle\rho, f\left(\frac{d f}{d t}+L f\right)\right\rangle+\langle\rho, g\rangle+\left\langle\frac{d \rho}{d t}-L \rho, f^{2}\right\rangle,
$$

for some $g \neq 0 \in H^{+}$.

At the time $T$, we are given a function $f_{0}$. We then construct $\sigma=$ $(\exp (T-t) L) f_{0}$. For this $f, \frac{d f}{d t}+L f=0$. Hence

$$
\frac{d}{d t}\left\langle\rho, f^{2}\right\rangle \geq\langle\rho, g\rangle \text {. }
$$

Let $T_{0}>0$ be the first time so that $\langle\rho, g\rangle>0$ for all $0 \leq t<T_{0}$ and for all $g \neq 0 \in H^{+}$. Our assumption says that this is true for $t=0$, hence $T_{0} \geq 0$. On the other hand, we can replace $\rho$ by $\rho+\varepsilon 1$ for small $\varepsilon>0$. In that case $T_{0}>0$. The above equation shows that $T_{0}$ can be prolonged to $T$.

We conclude with the following theorem:

Theorem A.2. Suppose $\rho_{0} \in \overline{H^{+}}$and $\frac{d \rho}{d t}-\Delta \rho \in \overline{H^{+}}$. Then if (A.12) holds, $\rho \in \overline{H^{+}}$for all $t$.

The weak maximum principle allows us to give estimate for the operator $\exp (t L)$. The idea is to find good super or sub solution of the heat equation and as a result, one finds estimate of solutions of the heat equation.

\section{A.4. Sobolev inequality and analytic dimension}

On a more abstract space that we are discussing, perhaps we can define Sobolev inequality by the following inequality:

For all $f \in \overline{H_{1}^{+}}$,

$$
\|f\|^{\frac{m+2}{m}} \leq c_{1}\|f\|_{1}\langle f, 1\rangle^{\frac{2}{m}}+c_{2}\|f\|\langle f, 1\rangle^{\frac{2}{m}},
$$

where $m, c_{1}$, and $c_{2}$ are positive constants independent of $f$. 
The smallest $m$ satisfying this inequality will be called the analytic dimension.

The heat kernel can be formulated in the following way: Take the tensor product $H \otimes H$ where we pick $\left\{e_{i}\right\}$ to be orthonormal basis for $H$ and $\left\{e_{i} \otimes\right.$ $\left.e_{j}\right\}$ for $H \otimes H \cdot \exp (t L)$ defines an element in $H \otimes H$ in the following way:

For any $f, g \in H,\langle\exp (t L) f, g\rangle$ defines a bilinear form and hence a linear functional on $H \otimes H$. By duality, it gives rise to an element $\rho \in H \otimes H$ so that

$$
\langle\exp (t L) f, g\rangle=\langle\rho, f \otimes g\rangle
$$

The element $\rho$ will satisfy the heat equation. The fact that $L$ is self-adjoint implies that $\rho$ is symmetric.

Theorem A.3. Assume Sobolev inequality (A.12) holds, then $\operatorname{tr} \exp (t L) \leq$ $C t^{-\frac{m}{2}}$ and the dimension of the space is not greater than $\mathrm{m}$.

Proof. Let $\left\{\varphi_{i}\right\}$ be an orthonormal base of $H$. Then $\sum_{i} \varphi_{i} \otimes \varphi_{i}$ can be considered as the Delta function.

The trace of $\exp (2 t L)$ is defined by

$$
\sum_{i}\left\langle\exp (2 t L) \varphi_{i}, \varphi_{i}\right\rangle
$$

which is equal to

$$
\sum_{i}\left\langle\exp (t L) \exp (t L) \varphi_{i}, \varphi_{i}\right\rangle=\sum\left\|\exp (t L) \varphi_{i}\right\|^{2} .
$$

Suppose we consider $\rho_{0}=\sum \varphi_{i} \otimes \varphi_{i}$ as element in $H \otimes H$, and $\exp (t L)$ acts on $H \otimes H$ through the action on the first factor. Then we have

$$
\rho_{t}=\sum_{i} \exp (t L) \varphi_{i} \otimes \varphi_{i}
$$

Hence

$$
\left\|\rho_{t}\right\|^{2}=\sum_{i}\left\|\exp (t L) \varphi_{i}\right\|^{2}
$$

Now

$$
\frac{d}{d t}\left\|\rho_{t}\right\|^{2}=2\left\langle\frac{d \rho_{t}}{d t}, \rho_{t}\right\rangle=-2\left\langle-L \rho_{t}, \rho_{t}\right\rangle
$$


and

$$
\frac{d}{d t}\left\langle\rho_{t}, 1 \otimes 1\right\rangle=\left\langle L \rho_{t}, 1 \otimes 1\right\rangle=0
$$

Hence

$$
\left\langle\rho_{t}, 1 \otimes 1\right\rangle=\left\langle\rho_{0}, 1 \otimes 1\right\rangle=1
$$

By (A.15), we find

$$
c_{1} \frac{d}{d t}\left\|\rho_{t}\right\| \leq-\left\|\rho_{t}\right\|^{\frac{m+2}{m}}+c_{2}\left\|\rho_{t}\right\|
$$

Hence, $\left\|\rho_{t}\right\| \leq\left[\frac{1}{m c_{1}}\left(\exp \left(\frac{2 c_{1}}{c_{2}} t\right)-1\right)\right]^{-\frac{m}{2}}$. The inequality in theorem (A.8) is proved.

However, we need to prove $\rho_{t} \in \overline{H_{1}^{+}}$. However, this can be achieved as in (A.11).

For comparison with classical argument, we reproduce the following argument of Nash [11]. Note that Sobolov inequality says that for any smooth function $f$,

$$
\left(\int f^{\frac{2 m}{m-2}}\right)^{\frac{m-2}{2 m}} \leq c_{1}\left(\int|\nabla f|^{2}\right)^{\frac{1}{2}}+c_{2}\left(\int f^{2}\right)^{\frac{1}{2}}
$$

where $c_{1}$ and $c_{2}$ are constants independent of $f$.

By applying Hölder inequality, we obtain

$$
\left(\int f^{2}\right) \leq\left(\int f^{\frac{2 m}{m-2}}\right)^{\frac{m-2}{m+2}}\left(\int|f|\right)^{\frac{4}{m+2}} .
$$

Hence

$$
\left(\int f^{2}\right)^{\frac{m+2}{2 m}} \leq\left(\int f^{\frac{2 m}{m-2}}\right)^{\frac{m-2}{2 m}}\left(\int|f|\right)^{\frac{2}{m}}
$$

and

$$
\begin{aligned}
\left(\int f^{2}\right)^{\frac{m+2}{2 m}} \leq & c_{1}\left(\int|\nabla f|^{2}\right)^{\frac{1}{2}}\left(\int|f|\right)^{\frac{2}{m}} \\
& +c_{2}\left(\int f^{2}\right)^{\frac{1}{2}}\left(\int|f|\right)^{\frac{2}{m}} .
\end{aligned}
$$


Let us define the number $m$ that satisfies (A.26) for all $f \in C$ to be analytic dimension of our triple $(H, C, L)$ (Note that $\int|\nabla f|^{2}=-\langle f, \Delta f\rangle$ ).

The semigroup $\exp (t L)$ acts on $H=L^{2}(M)$ with a kernel function $h(t, x, y)$, which satisfies the heat equation

$$
\left\{\begin{array}{l}
\frac{\partial h}{\partial t}=\Delta_{x} h(t, x, y) \\
\lim _{t \rightarrow 0} h(t, x, y)=\delta_{y}(x)
\end{array}\right.
$$

The integral $\int h(t, x, y) d y$ is preserved by the first equation. Since $\lim _{t \rightarrow 0} \int h(t, x, y) d y=1$, we conclude that for all $t>0$,

$$
\int h(t, x, y) d y=1
$$

One can also prove that $\frac{d}{d t} \int|h| \leq 0$. Hence, $\int|h|=1$ which means $h(t, x, y) \geq 0$.

Note that

$$
\frac{d}{d t} \int h^{2}=2 \int h \Delta h=-2 \int\left|\nabla_{x} h\right|^{2}
$$

Hence

$$
\frac{d}{d t} \int h^{2} \leq-C_{3}\left(\int h^{2}\right)^{\frac{m+2}{m}}+C_{4} \int h^{2}
$$

Since

$$
\lim _{t \rightarrow 0} \int h^{2}(t, x, y)=\infty,
$$

we conclude that for $t$ small,

$$
\int h^{2}(t, x, y) \leq C^{\prime}\left(1-\mathrm{e}^{-c_{4} t}\right)^{\frac{m}{2}}
$$

The semigroup property of $\exp (-t L)$ shows

$$
\begin{aligned}
h(2 t, x, x) & =\int h(t, x, y) h(t, y, x) \\
& =\int h^{2}(t, x, y) .
\end{aligned}
$$


Hence

$$
\begin{aligned}
h(t, x, y) & \leq \bar{C}\left(1-\mathrm{e}^{-\frac{c_{4}}{2} t}\right)^{\frac{m}{2}}, \\
\operatorname{Tr} \exp (t \Delta) & =\int h(t, x, x) d x \\
& \leq \bar{C}\left(1-\mathrm{e}^{-\frac{c_{4}}{2} t}\right)^{\frac{m}{2}} \operatorname{vol}(M) .
\end{aligned}
$$

Since we assume

$$
\operatorname{Tr} \exp (t \Delta) \sim c t^{\frac{-n}{2}}
$$

we conclude that the analytic dimension $m$ is not less than the dimension of the space.

\section{A.5. Compactness}

Before we discuss compactness, we need to introduce a norm on the algebra $H_{1}$, the completion of $C$ by the norm $-\left\langle\varphi_{1}, L \varphi\right\rangle$.

The map

$$
\begin{aligned}
H_{1} \bigotimes H_{1} & \longrightarrow H_{1}, \\
f \otimes g & \longrightarrow f g
\end{aligned}
$$

is assumed to be continuous bilinear and there is a positive constant $B$, so that

$$
\|f g\|_{1} \leq B\|f\|_{1}\|g\|_{1}
$$

for all $f, g \in H_{1}$. The smallest constant $B$ will be defined to be the norm of the algebra $H_{1}$.

Let us fix one triple $(H, C, L)$ and we assume that we have a family of such triples such that the constant $B$ in (A.37) is uniform for all triples in this family.

Theorem A.4. A family of triples is precompact if within the family, the constant B, constants $c_{1}$ and $c_{2}$ in the Sobolov inequality (A.13) and the constant $\overline{\bar{\lambda}}_{R}$ defined by (A.5) are uniformly bounded.

Proof. The Sobolov inequality gives lower estimate of eigenvalues in the family which (A.5) gives upper estimate. Hence, we can take limit of the 
eigenvalues and eigenvectors of subsequence of the operators to form a new operator.

\section{A.6. Heat equations}

First of all, we note that for any $f, \rho \in C$,

$$
\left\langle\rho, L\left(e^{f}\right)=\sum_{m} \frac{1}{m !}\left\langle\rho, L\left(f^{m}\right)\right\rangle .\right.
$$

However,

$$
\left\langle\rho, L\left(f^{m}\right)\right\rangle=\left\langle\rho, f L\left(f^{m-1}\right)\right\rangle+\left\langle\rho, f^{m-1} L(f)\right\rangle+2\left\langle\rho d\left(f^{m-1}\right), d f\right\rangle
$$

By induction, we obtain

$$
\left\langle\rho, L\left(f^{m}\right)\right\rangle=m\left\langle\rho f^{m-1}, L(f)\right\rangle+m(m-1)\left\langle\rho f^{m-2} d f, d f\right\rangle .
$$

In particular,

$$
\left\langle\rho, L\left(e^{f}\right)\right\rangle=\left\langle\rho e^{f}, L f\right\rangle+\left\langle\rho e^{f} d f, d f\right\rangle
$$

For functions $g_{1}(t), g_{2}(t), a_{0}(x, y)$ and $a_{1}(x, y)$, we define

$$
F=g_{1}(t) \exp \left(\frac{-f}{g_{2}(t)}\right)\left(a_{0}+a_{1} t\right) .
$$

Then we have

$$
\begin{aligned}
\left\langle\rho,\left(\frac{\partial}{\partial t}-L\right) F\right\rangle= & \left\langle\rho\left(\frac{g_{1}^{\prime}}{g_{1}}+\frac{g_{2}^{\prime} f}{g_{2}^{2}}-\frac{L(f)}{g_{2}}\right), F\right\rangle-\left\langle\rho \frac{d f}{g_{2}^{2}}, F d f\right\rangle \\
& +\left\langle\rho \frac{d f}{g_{2}\left(a_{0}+a_{1} t\right)}, F\left[d a_{0}+\left(d a_{1}\right) t\right]\right\rangle+\left\langle\frac{\rho a_{1}}{a_{0}+a_{1} t}, F\right\rangle \\
& -\left\langle\rho \frac{L\left(a_{0}\right)+L\left(a_{1}\right) t}{a_{0}+a_{1} t}, F\right\rangle .
\end{aligned}
$$

In order to solve the heat equations, we require that for $t$ small,

$$
\left|\left\langle\frac{g_{1}^{\prime}}{g_{1}}-\frac{L(f)}{g_{2}}-\frac{\left\langle d f, d a_{0}\right\rangle}{g_{2} a_{0}}, F \rho\right\rangle\right| \leq C t\|\rho\|,
$$




$$
\left|\left\langle\frac{g_{2}^{\prime} f}{g_{2}^{2}}-\frac{\langle d f, d f\rangle}{g_{2}^{2}}, F \rho\right\rangle\right| \leq C t\|\rho\|
$$

$$
\left|\left\langle a_{1}-L\left(a_{0}\right)-\frac{t}{g_{2}}\left\langle d f, d a_{1}\right\rangle+\frac{t}{g_{2}} \frac{a_{1}\left\langle d f, d a_{0}\right\rangle}{a_{0}}, F \rho\right\rangle\right| \leq C t\|\rho\|,
$$

where $C$ is a constant independent of $t$.

Typically, we choose

$$
g_{1}(t)=\alpha_{1} t^{-n / 2}
$$

and

$$
g_{2}(t)=\alpha_{2} t
$$

where $\alpha_{1}$ and $\alpha_{2}$ are constants to be chosen so that

$$
\lim _{t \rightarrow 0}\left\langle 1, g_{1}(t) \exp \left(\frac{-f}{g_{2}(t)}\right)\right\rangle=1
$$

and we like to choose $f \in H \otimes H$, so that

$$
\lim _{t \rightarrow 0}\left\langle\rho, \alpha_{1} t^{-n / 2} \exp \left(\frac{-f(x, y)}{\alpha_{2} t}\right)\right\rangle=\rho(y) .
$$

For Riemannian manifold, $f(x, y)$ is $d^{2}(x, y)$ up to lower-order term where $d(x, y)$ is the distance between $x$ and $y$.

Suppose $f$ satisfies (A.43), (A.44), (A.45) and (A.48). Then by (A.42), the operator $\frac{\partial}{\partial t}-L F$ satisfies the following:

$$
\left\|\left(\frac{\partial}{\partial t}-L\right) F\right\| \leq C t
$$

We claim that as an operator,

$$
\left\|F^{-1}\left(\frac{\partial}{\partial t}-L\right) F\right\| \leq C^{\prime} t .
$$

In fact, we have

$$
F^{-1}\left(\frac{\partial}{\partial t}-L\right) F \varphi=\psi
$$


Then

$$
\|\psi\|^{2}=\|F(\psi)\|^{2}-2 \int_{0}^{t}\left\langle\frac{\partial}{\partial s} F(\psi), F(\psi)\right\rangle .
$$

However,

$$
\|F(\psi)\|^{2}=\left\|\left(\frac{\partial}{\partial t}-L\right) F \varphi\right\|^{2} \leq C^{2} t^{2}\|\varphi\|^{2} .
$$

and

$$
\text { (A.55) }\left\langle\frac{\partial}{\partial s} F(\psi), F(\psi)\right\rangle=\langle L(F(\psi)), F(\psi)\rangle+\left\langle\left(\frac{\partial}{\partial s}-L\right) F(\psi), F(\psi)\right\rangle
$$

Since

$$
\langle L(F(\psi)), F(\psi)\rangle \leq 0
$$

we obtain

$$
\begin{aligned}
& \|\psi\|^{2} \leq C^{2} t^{2}\|\varphi\|^{2}+C^{2} t^{2}\|\psi\|\|\varphi\| \\
& \text { which implies }\|\psi\|^{2} \leq C^{\prime \prime} t^{2}\|\varphi\|^{2}
\end{aligned}
$$

By requiring further constrains on $f$, one can find condition depending only on $L^{i}(f)$ for the existence of the asymptotic expansion of the $t^{n / 2} \operatorname{tr} \exp (t L)$.

Let $E(t L)=\exp (t L)$ and $F(t)$ be any one parameter family of operators such that $F(0)$ is identity and

(A.58) $\left\|F^{-1}\left(\frac{\partial}{\partial t}-L\right) F\right\|^{2}=\sup _{\|\psi\| \leq 1}\left\|F^{-1}\left(\frac{\partial}{\partial t}-L\right) F(\psi)\right\|^{2} \leqslant c t^{k}$, 
for some $k \geqslant 0$. Then

$$
\begin{aligned}
E(t L)-F(t)= & \int_{0}^{t} \frac{d}{d s}(E(s L) F(t-s)) \\
= & \int_{0}^{t} E(s L)\left(L-\frac{\partial}{\partial s}\right) F \\
= & \int_{0}^{t} E(s L) F(t-s) F^{-1}(t-s)\left(L-\frac{\partial}{\partial s}\right) F \\
= & \int_{0}^{t} E(s L)(F(t-s)-E(t-s)) F^{-1}(t-s)\left(L-\frac{\partial}{\partial s}\right) F \\
& +E(t L) \int_{0}^{t} F^{-1}\left(L-\frac{\partial}{\partial s}\right) F .
\end{aligned}
$$

Define $\mathcal{F}=F^{-1}\left(\frac{\partial}{\partial t}-L\right) F$. Then

$$
\begin{aligned}
E(t L)-F(t)= & E(t L)\left\{\int_{0}^{t} \mathcal{F} \pm \iint_{s_{1}+s_{2}=1} \mathcal{F}\left(s_{1}\right) \mathcal{F}\left(s_{2}\right)\right. \\
& \left. \pm \iint_{s_{1}+s_{2}+s_{3}=1} \mathcal{F}\left(s_{1}\right) \mathcal{F}\left(s_{2}\right) \mathcal{F}\left(s_{3}\right) \pm \cdots\right\} \\
= & E(t L) \widetilde{\mathcal{F}}(t)=(E(t L)-F(t)) \widetilde{\mathcal{F}}(t)+F(t) \widetilde{\mathcal{F}}(t) .
\end{aligned}
$$

Hence

$$
E(t L)-F(t)=F(t) \widetilde{\mathcal{F}}(t)(1-\widetilde{\mathcal{F}}(t))^{-1}
$$

Now let $\psi_{\lambda}$ be an eigenfunction of $L$ with eigenvalue $-\lambda$. Then

$$
\begin{aligned}
\left\|F(t) \psi_{\lambda}\right\| & \leq\left\|F(t)-E(t L) \psi_{\lambda}\right\|+\left\|E(t L) \psi_{\lambda}\right\| \\
& \leq c t^{k+1}\left\|F(t) \psi_{\lambda}\right\|+\mathrm{e}^{-t \lambda} .
\end{aligned}
$$

Hence,

$$
\begin{gathered}
\left|\left\langle E(t L) \psi_{\lambda}, \psi_{\lambda}\right\rangle-\left\langle F(t) \psi_{\lambda}, \psi_{\lambda}\right\rangle\right| \leq c t^{k+1}\left\|F(t) \psi_{\lambda}\right\| \\
\leq \frac{c t^{k+1}}{1-c t^{k+1}} \mathrm{e}^{-t \lambda} .
\end{gathered}
$$


Since $\left\{\psi_{\lambda}\right\}$ form an orthonomal basis for $H$, we obtain

$$
\left(1-c t^{k+1}\right) \operatorname{tr} F(t) \leq \operatorname{tr} E(t L) \leq\left(1-c t^{k+1}\right)\left(1-2 c t^{k+1}\right)^{-1} \operatorname{tr} F .
$$

Theorem A.5. Given a self-adjoint operator L operating on a Hilbert space $H$ so that $\exp (t L)$ is of trace class. Suppose there is also a one parameter of operators $F(t)$, for $t>0$, which is also of trace class and satisfied the following conditions:

$$
F(0)=\text { identity, }\left\|F^{-1}\left(\frac{\partial}{\partial t}-L\right) F\right\| \leq c t^{k} .
$$

Then

(A.66) $\quad\left(1-c t^{k+1}\right) \operatorname{tr} F(t) \leq \operatorname{tr} \exp (t L) \leq\left(1-c t^{k+1}\right)\left(1-2 c t^{k+1}\right)^{-1} \operatorname{tr} F$.

Theorem A.6. On our triple, if we can find $f$ in the tensor product of $H$ with itself, that satisfies (A.48), (A.49) and also (A.43), (A.44), (A.45). Then we can define a one parameter family of operators $F(t)$, for $t>0$, so that the hypothesis (A.65) holds and therefore (A.66) holds. The asymptotic of the heat operator therefore holds up to second order.

Note that in principle, we can go to any order for the asymptotic of the heat operator as long as we know the information of the action of high power of $L$ acting on $f$.

\section{A.7. Hodge theory for differential forms}

Let us illustrate the ideas of Hodge Theory for one-forms and two-forms. We shall call our space to be finite type if there are finite number of one-forms $\theta_{i}$ generating the space of one-forms over $C$ and

$$
\left\langle\theta_{i}, \theta_{j}\right\rangle=\rho_{i} \delta_{i j}
$$

This can be achieved for any smooth manifold by partition of unity.

Then for any one-form $\omega$, there are $f_{i} \in C$, so that

$$
\omega=\sum_{i} f_{i} \theta_{i}
$$

Let

$$
\theta_{i}=\sum a_{i j} d g_{j}
$$


Then for any $h \in C$,

$$
\begin{aligned}
\langle\delta \omega, h\rangle & =\sum_{i} f_{i j}\left\langle d g_{j}, d h\right\rangle \\
& =\frac{1}{2} \sum f_{i} a_{i j}\left(L\left(g_{j} h\right)-g_{j} L(h)-h L\left(g_{i}\right)\right) \\
& =\frac{1}{2} \sum_{i} g_{j} L\left(f_{i} a_{i j}\right) h-\frac{1}{2} \sum_{i} L\left(f_{i} a_{i j} g_{j}\right) h-\frac{1}{2} \sum_{i}\left(f_{i} a_{i j} L\left(g_{i}\right)\right) h \\
(\mathrm{~A} .69) & =-\sum_{i}\left\langle d\left(f_{i} a_{i j}\right\rangle, d g_{j}\right\rangle h-\sum_{i} f_{i} a_{i j} L\left(g_{i}\right) h .
\end{aligned}
$$

We conclude

$$
\delta \omega=-\left\langle d f_{i}, \theta_{i}\right\rangle-\sum_{i} f_{i}\left(\left\langle d a_{i j}, d \theta_{j}\right\rangle+a_{i j} L\left(g_{j}\right)\right) .
$$

Now let us discuss two-forms

$$
\Omega=\sum b_{i j} \theta_{i} \wedge \theta_{j},
$$

where $b_{i j}=-b_{j i}$. Then

$$
\begin{aligned}
\langle\delta \Omega, \omega\rangle & =\left\langle\Omega, \sum d f_{i} \wedge \theta_{i}+\sum f_{i} d \theta_{i}\right\rangle \\
& =\left\langle\Omega, \sum f_{i, j} \theta_{j} \wedge \theta_{i}+\sum_{i} f_{i} d \theta_{i}\right\rangle \\
& =\left\langle\Omega, \frac{1}{2} \sum\left(f_{i, j}-f_{j, i}\right) \theta_{j} \wedge \theta_{i}+\sum_{i} f_{i} d \theta_{i}\right\rangle \\
& =\frac{1}{2} \sum\left(f_{j, i}-f_{i, j}\right) b_{i j} \rho_{i} \rho_{j}+\left\langle\Omega, \sum f_{i} d \theta_{i}\right\rangle .
\end{aligned}
$$

Let

$$
\omega^{\prime}=\sum f_{j} b_{i j} \rho_{i} \rho_{j} \theta_{i}
$$

We have

$$
\begin{aligned}
\left\langle\delta \omega^{\prime}, 1\right\rangle= & -\sum\left\langle d\left(f_{j} b_{i j} \rho_{j}\right), \theta_{i}\right\rangle-\sum f_{i} b_{i j} \rho_{j}\left(\left\langle d a_{i k}, d g_{k}\right\rangle+a_{i k} L\left(g_{k}\right)\right) \\
= & -\sum f_{j, i} b_{i j} \rho_{j} \rho_{i}-\sum f_{j}\left(b_{i j} \rho_{j}\right)_{, i} \rho_{i} \\
& -\sum f_{i} b_{i j} \rho_{j}\left(\left\langle d a_{i k}, d g_{k}\right\rangle+a_{i k} L\left(g_{k}\right)\right) .
\end{aligned}
$$


Since $\left\langle\delta \omega^{\prime}, 1\right\rangle=0$ and

$$
\sum\left(f_{j, i}-f_{i, j}\right) b_{i j} \rho_{i} \rho_{j}=2 \sum f_{j, i} b_{i j} \rho_{i} \rho_{j},
$$

we conclude

$$
\begin{aligned}
\langle\delta \Omega, \omega\rangle= & -\sum f_{j}\left(b_{i j} \rho_{j}\right)_{, i} \rho_{i} \\
& -\sum f_{i} b_{i j} \rho_{j}\left(\left\langle d a_{i k}, d g_{k}\right\rangle+a_{i k} L\left(g_{k}\right)\right)+\left\langle\Omega, \sum f_{i} d \theta_{i}\right\rangle
\end{aligned}
$$

and

$$
\begin{aligned}
\delta \Omega= & -\sum\left(b_{i j} \rho_{j}\right)_{, i} \rho_{i} \rho_{j}^{-1} \theta_{j}+\sum b_{i j} \rho_{i}\left(\left\langle d a_{j k}, d g_{k}\right\rangle\right. \\
& \left.+a_{j k} L\left(g_{k}\right)\right) \rho_{j}^{-1} \theta_{j}-\sum\left\langle\Omega, d \theta_{i}\right\rangle \rho_{i}^{-1} \theta_{i}
\end{aligned}
$$

Let us now look at the Bochner form for one-form

$$
\begin{aligned}
\|d \omega\|^{2}+\|\delta \omega\|^{2}= & \left\|\frac{1}{2} \sum\left(f_{i, j}-f_{j, i}\right) \theta_{i} \wedge \theta_{j}+\sum f_{i} d \theta_{i}\right\|^{2} \\
& +\left\|\sum\left\langle d f_{i}, \theta_{i}\right\rangle+\sum f_{i}\left(\left\langle d\left(a_{i j}\right), d g_{j}\right\rangle+a_{i j} L\left(g_{j}\right)\right)\right\|^{2} \\
= & \frac{1}{2} \sum\left(f_{i, j}-f_{j, i}\right)^{2} \rho_{i} \rho_{j}+\sum\left(f_{i, j}-f_{j, i}\right) f_{i}\left\langle\theta_{i} \wedge \theta_{j}, d \theta_{i}\right\rangle \\
& +\sum f_{i} f_{j}\left\langle d \theta_{i}, d \theta_{j}\right\rangle+\left(\sum_{i} f_{i, i} \rho_{i}\right)^{2} \\
& +2\left(\sum f_{i, i} \rho_{i}\right) \sum f_{k}\left(\left\langle d\left(a_{k j}\right), d g_{j}\right\rangle+a_{k j} L\left(g_{j}\right)\right) \\
& +\left\|\sum f_{i}\left(\left\langle d\left(a_{i, j}\right), d g_{j}\right\rangle+a_{i, j} L\left(g_{j}\right)\right\rangle\right\|^{2} .
\end{aligned}
$$

Define one-form

$$
\omega^{\prime \prime}=\sum f_{i} f_{j, i} \rho_{i} \theta_{j}
$$

we have $\left\langle\delta \omega^{\prime \prime}, 1\right\rangle=0$. Hence

$$
\sum\left(f_{i} f_{j, i} \rho_{i}\right)_{j} \rho_{j}+\sum f_{i} f_{j, i} \rho_{i}\left(\left\langle d a_{j k}, d g_{k}\right\rangle+a_{j k} L\left(g_{k}\right)\right)=0
$$

and

$$
\begin{aligned}
-\sum f_{i, j} f_{j, i} \rho_{i} \rho_{j}= & \sum f_{i} f_{j, i j} \rho_{i} \rho_{j}-\sum f_{i} f_{j, i} \rho_{i, j} \rho_{j} \\
& +\sum f_{i} f_{j, i} \rho_{k}\left(\left\langle d a_{j k}, d g_{k}\right\rangle+a_{j k} L\left(g_{k}\right)\right) .
\end{aligned}
$$


Similarly, let

$$
\omega^{\prime \prime \prime}=\sum f_{i} f_{j, j} \rho_{i} \rho_{j} \theta_{i}
$$

Then $\left\langle\delta \omega^{\prime \prime \prime}, 1\right\rangle=0$ and

$$
\sum\left(f_{i} f_{j, j} \rho_{j}\right)_{i} \rho_{i}+\sum f_{i} f_{j, j} \rho_{j}\left(\left\langle d a_{i k}, d g_{k}\right\rangle+a_{i k} L\left(g_{k}\right)\right)=0
$$

Hence,

$$
\begin{aligned}
\sum f_{i} f_{j, j i} \rho_{j} \rho_{i}= & -\left(\sum_{i} f_{i, i} \rho_{i}\right)^{2}-\sum f_{i} f_{j, j} \rho_{j, i} \rho_{i} \\
& +\sum f_{i} f_{j, j} \rho_{j}\left(\left\langle d a_{i k}, d g_{k}\right\rangle+a_{i k} L\left(g_{k}\right)\right) .
\end{aligned}
$$

$$
\begin{aligned}
\|d \omega\|^{2}+\|\delta \omega\|^{2}= & \sum f_{i, j}^{2} \rho_{i} \rho_{j}+\sum\left(f_{i, j}-f_{j, i}\right) f_{i}\left\langle\theta_{i} \wedge \theta_{j}, d \theta_{i}\right\rangle \\
& +\sum f_{i} f_{j}\left\langle d \theta_{i}, d \theta_{j}\right\rangle-\sum f_{i} f_{j, i} \rho_{i, j} \rho_{j} \\
& +\sum f_{i} f_{j, i} \rho_{i}\left(\left\langle d a_{j k}, d g_{k}\right\rangle+a_{j k} L\left(g_{k}\right)\right) \\
& -\sum f_{i} f_{j, j} \rho_{j, i} \rho_{i}+\sum f_{i} f_{j, j} \rho_{j}\left(\left\langle d a_{i k}, d g_{k}\right\rangle+a_{i k} L\left(g_{k}\right)\right) \\
& +2 \sum f_{i, i} \rho_{i} \sum f_{k}\left(\left\langle d\left(a_{k j}\right), d g_{j}\right\rangle+a_{k j} L\left(g_{j}\right)\right) \\
& +\left\|\sum f_{i}\left(\left\langle d a_{i j}, d g_{j}\right\rangle+a_{i j} L\left(g_{j}\right)\right)\right\|^{2} .
\end{aligned}
$$

From this formula, we can put terms together to form the Bochner mentioned in Section 6. It allows us to prove the corresponding Sobolev inequality (A.8) for forms from Sobolev inequality for functions. Hence, we can control the growth of the spectrum of Laplacian acting on forms and their eigenspaces are all finite-dimensional.

\section{A.8. Star operators}

The operator manifold may not have Poincaré duality and hence we may not have star operator in ordinary sense. However, we still can define them to be two endomorphisms $*$ and $*^{\prime}$ from the exterior algebra of de Rham 
forms to itself, which satisfy the following two identities:

$$
d *=*^{\prime} \delta, \quad * d=\delta *^{\prime} .
$$

In this way, if such operators exist, a coclosed form is mapped under $*$ to be a closed form. And a closed form is mapped under $*^{\prime}$ to be coclosed. The structure of $*^{2}, * *^{\prime}, *^{\prime} *$, or $\left(*^{\prime}\right)^{2}$ would be interesting to understand. It is related to Poincaré duality. For example, if $*=*^{\prime}$ and $*^{2}$ is invertible, we have Poincaré duality. Otherwise, their kernel and cokernel would have suitable duality.

\section{A.9. Examples}

An important class of examples of singular varieties are given by metric simplicial complex which consist of several smooth manifolds $M_{i}$ glued together. Each $M_{i} \backslash\left(\cup_{j \neq i}\left(M_{j}\right)\right)$ would be smooth with a smooth metric whose metric completion is compact.

Let $C$ be the space of continuous functions which are continuous on $\cup_{i} M_{i}$, smooth on $M_{i} \backslash\left(\cup_{j \neq i} M_{j}\right)$ for all $i$ and satisfy the following condition: For each $M_{i}$, let $M_{j_{1}}, \cdots, M_{j_{i}}$ be the set so that

$$
M_{i} \subset \bar{M}_{j_{\ell}}
$$

and no other $M_{k}$ with

$$
M_{i} \subset \bar{M}_{k} \subset \bar{M}_{j_{\ell}}
$$

For each $j_{\ell}, M_{i}$ has a neighborhood defined by a tube $N_{j_{\ell}}(r)$ of radius $r$. Along $\partial N_{j_{\ell}}(r)$, there is an outer normal $\nu_{j_{\ell}}$ and we require that for all $M_{i}$,

$$
\lim _{r \rightarrow 0} \sum_{\ell} \int_{\partial N_{j_{\ell}}(r)} \nu_{j_{\ell}}(f)=0 .
$$

Note that $\Delta f$ can be defined in the sense of distribution on $M_{i}$ by the formula: For any function $g \in C$, which can be written as a function of a distance function $r(x)$ from a point $x$, we can define $\Delta g$ on each $M_{i}$ which contains $x$ when $r(x)$ is small. For such function $g$, we require the following 
formula to hold:

$$
\begin{aligned}
\sum_{\ell} \int_{N_{j_{\ell}}(r)} g(\Delta f)=\sum_{\ell} \int_{N_{j_{\ell}}(r)}(f) \Delta g+\sum_{\ell} \int_{\partial N_{j_{\ell}}(r)} g \nu_{j_{\ell}}(f) \\
-\sum_{\ell} \int_{\partial N_{j_{\ell}}(r)} f \cdot \nu_{j_{\ell}}(g) .
\end{aligned}
$$

We define the Hilbert space to be the direct sum $\bigoplus L^{2}\left(M_{i}\right)$. Note that the eigenfunctions or the heat kernel may not be an element in $C$.

The triple defined in this manner is interesting even for the configuration of two circles joining at one point.

Consider an embedded subvariety defined by a function $h$. Let $h \in C$ be in our triple. We can define a new triple where the algebra of smooth functions would be given by $C$ quotiented by $h C$. The new Hilbert space is the completion of $C / h C$ given by the norm

$$
\left\langle f+h f^{\prime}, g+h g^{\prime}\right\rangle=\lim _{t \rightarrow 0} \frac{t^{-1 / 2}}{2 \sqrt{\pi}}\left\langle e^{-\frac{h^{2}}{4 t}}\left(f+h f^{\prime}\right),\left(g+h f^{\prime}\right)|d h|\right\rangle .
$$

The self-adjoint operator is defined by the inner product coming from the norm

$$
\left\|f+h f^{\prime}\right\|_{1}^{2}=\inf _{f^{\prime}} \lim _{t \rightarrow 0} \frac{t^{-1 / 2}}{2 \sqrt{\pi}}\left\langle e^{-\frac{h^{2}}{4 t}}\left(d f+d\left(h f^{\prime}\right)\right),\left(d f+d\left(h f^{\prime}\right)\right)|d h|\right\rangle .
$$

When the triple comes from a smooth manifold and zero is not a critical value of $h$, then the new triple corresponds to the smooth manifold define by $h=0$.

\section{References}

[1] M. Atiyah, R. Bott and V. K. Patodi, On the heat equation and the index theorem, Invent. Math. 19(1973), 279-330.

[2] D. Bakry and M. Emery, Diffusions hypercontractives, Sem. de Prob.XIX, 177-206, Lecture Notes in Math. 1123, Springer-Verlag 1985.

[3] E. Calabi, The space of Kähler metrics, Proc. Int. Congress Math. Amsterdam, 1954, Vol. 2, pp. 206-207.

[4] Fan Chung and S.-T. Yau, Logarithmic Harnack inequalities, Math. Res. Lett. 3(6) (1996), 793-812. 
[5] A. Connes, Noncommutative geometry. Academic Press, San Diego, CA, 1994.

[6] G.W. Gibbons and S.W. Hawking, Action integrals and partition functions in quantum Gravity, Phys. Rev. D 15(1977), 2752-2756.

[7] A, Grigor'yan, Y. Lin, Y. Moranov and S.-T. Yau, Homologies of digraph, arXiv:1207.2834.

[8] W.V.D. Hodge, The theory and applications of harmonic integrals, 2nd ed. Cambridge University Press, Cambridge, 1952. x+282 pp.

[9] Y. Lin and S.-T. Yau, Ricci curvature and eigenvalue estimate on locally finite graphs, Math. Res. Lett. 17(2) (2010), 343-356.

[10] Y. Lin, L. Lu and S.-T. Yau, Ricci curvature of graphs, Tohoku Math. J. (2) 63(4) (2011), 605-627.

[11] J. Nash, The imbedding problem for Riemannian manifolds, Ann. Math. (2) 63(1956), 20-63.

[12] Y. Ollivier, Ricci curvature of Markov chains on metric spaces, J. Funct. Anal. 256(3) (2009), 810-864.

[13] B. Riemann, Gesammelte mathematische Werke, wissenschaftlicher Nachlass und Nachträge. (German) [Collected mathematical works, scientific Nachlass and addenda] Edited and with a preface by Raghavan Narasimhan. Springer-Verlag, Berlin; BSB B. G. Teubner Verlagsgesellschaft, Leipzig, 1990. vi+911 pp.

[14] S.-T. Yau, On the Ricci curvature of a compact Kähler manifold and the complex Monge-Ampère equation I, Commun. Pure Appl. Math. 31(3) (1978), 339-411.

[15] S.-T. Yau, (ed.) Mirror symmetry I. AMS/IP Studies in Advanced Mathematics, 9, International Press, Cambridge, 1998.

Department of Mathematics

HARVARD UNIVERSITY

1 Oxford Street

CAmbridge, MA 02138

USA

E-mail address: yau@math.harvard.edu

Received OCtober 18, 2012 